\title{
Autoimmune Primary Adrenal Insufficiency
}

National Cancer Institute

\section{Source}

National Cancer Institute. Autoimmune Primary Adrenal Insufficiency. NCI Thesaurus.

Code C113814.

Diminished production of adrenocortical hormones due to autoimmune destruction of the adrenal glands. 